\title{
Acoustic search for high-energy neutrinos in the Lake Baikal: Results and plans
}

\author{
${ }^{a}$ Institute for Nuclear Research, Moscow, Russia \\ ${ }^{\mathrm{b}}$ Applied Physics Institute of Irkutsk State University, Gagarin blvd. 20, Irkutsk, Russia \\ ' Skobeltsyn Institute of Nuclear Physics MSU, Moscow, Russia \\ d Joint Institute for Nuclear Research, Dubna, Russia \\ e DESY, Zeuthen, Germany \\ ${ }^{\mathrm{f}}$ Nizhni Novgorod State Technical University, Nizhni Novgorod, Russia \\ ${ }^{\mathrm{g}}$ St. Petersburg State Marine University, St. Petersburg, Russia \\ ${ }^{\mathrm{h}}$ Kurchatov Institute, Moscow, Russia \\ i Institute of Solar-Terrestrial Physics, Lermontov st. 126a, Irkutsk, Russia
}

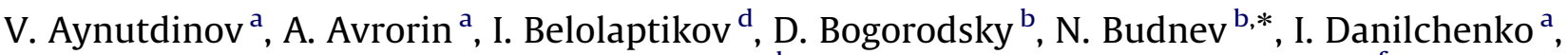
G. Domogatsky ${ }^{a}$, A. Doroshenko ${ }^{a}, A$. Dyachok ${ }^{b}, Z^{2 h .-A . ~ D z h i l k i b a e v ~}{ }^{a}$, S. Fialkovsky ${ }^{f}$, O. Gaponenko ${ }^{a}$, K. Golubkov ${ }^{\text {d }}$, O. Gress ${ }^{\text {b }}$, T. Gress ${ }^{\text {b }}$, O. Grishin ${ }^{\text {b }}$, V. Karnaukhov ${ }^{c}$, A. Klabukov ${ }^{\text {a }}$, A. Klimov ${ }^{\text {h }}$, A. Kochanov ${ }^{\text {, }}$, K. Konischev ${ }^{\text {d }}$, A. Korobchenko ${ }^{\text {b }}$, A. Koshechkin ${ }^{\text {, }}$, D. Kostunin ${ }^{\text {b }}$, V. Kulepov ${ }^{f}$, D. Kuleshov ${ }^{\text {a }}$, L. Kuzmichev ${ }^{c}$,

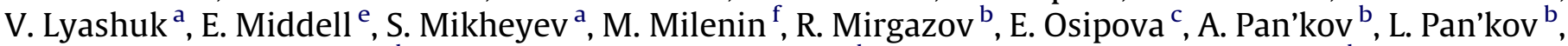
A. Panfilov ${ }^{a}$, A. Perevalov ${ }^{b}$, D. Petukhov ${ }^{a}$, E. Pliskovsky ${ }^{d}$, V. Poleschuk ${ }^{a}$, I. Portyanskaya $^{b}$, E. Popova $^{c}$,

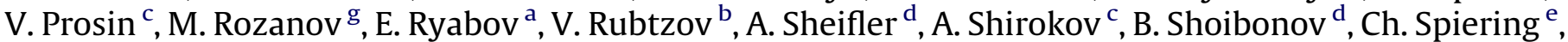
O. Suvorova $^{\text {a }}$, B. Tarashansky ${ }^{\text {b }}$, R. Wischnewski ${ }^{\text {e }}$, A. Zagorodnikov ${ }^{\text {b }}$, V. Zhukov $^{\text {a }}$, A. Yagunov $^{\text {b }}$ I. Yashin $^{\text {c }}$

\section{A R T I C L E I N F O}

Available online 14 December 2010

Keywords:

Baikal neutrino telescopes

Acoustic neutrino detection

\begin{abstract}
A B S T R A C T
We report the present status and perspectives of the feasibility study to detect cosmic neutrinos acoustically in the Lake Baikal. The results of background studies are presented. It was shown that most of the detected neutrino-like pulses come from the lake surface. This fact has been used in the project of an acoustic prototype detector that consists of compact Acoustic Sensor Modules with 4-channel antennas each, arranged above the Baikal Neutrino Telescope at shallow depths and "listening" the deep-water layers of the lake.
\end{abstract}

(c) 2010 Elsevier B.V. All rights reserved.

\section{Introduction}

Fifty years have passed since M. Markov proposed to build an optical Cherenkov array in ocean to search for high energy astrophysical neutrinos [1]. In 1998 the first underwater neutrino Cherenkov detector NT200 was constructed in the lake Baikal $[2,3]$. Other large scale Cherenkov neutrino telescopes AMANDA/IceCube [4] at the South Pole, ANTARES [5] in the Mediterranean Sea were put in operation later. The Baikal collaboration follows since several years a $R \& D$ program for a $\mathrm{km}^{3}$ scale water detector (Baikal-GVD) in the Lake Baikal [6]. The most recent milestones of the project were the construction and installation of new technology prototype strings in 2008-2010 years [7]. The main scientific goal of IceCube, Baikal-GVD and other $\mathrm{km}^{3}$ volume detectors is to search for high energy astrophysical neutrinos. Other topics include the indirect search for dark matter by searching for neutrinos produced in

\footnotetext{
* Corresponding author.

E-mail address: nbudnev@api.isu.ru (N. Budnev).
}

WIMP annihilation in the Sun or in the center of the Earth. The $\mathrm{km}^{3}$ volume detectors can also be used to search for exotic particles like magnetic monopoles, super-symmetric Q-balls or nuclearites.

The volume of the detectors to search for UHE astrophysical neutrinos should be probably much more than one cubic kilometer and far beyond the limits of reasonable size for an optical Cherenkov underwater/underice array. Back in 1957, Askaryan has shown that a high-energy particle cascade in water, besides the Cherenkov radiation, should also produce an acoustic signal [8]. The potential of the acoustic neutrino detection $[9,10]$ is based on the fact that the absorption length for acoustic waves in the frequency range of 5-30 kHz (the optimal range for acoustic signals from a shower) in sea water is at least an order of magnitude larger than that of Cherenkov radiation. In the fresh Baikal water this ratio is even close to 100 [11]. The second fact that is favorable for the detection of acoustic signals from cascade showers at distances of hundreds of meters, or even at such long distances as several kilometers, is that the amplitude of pulses produced by showers in the near-field zone decreases only as the square root of distance, while in the far-field zone it decreases, as the reciprocal of distance 
from the shower $[9,10]$. Therefore, in principle, a deep-water acoustic detector of high-energy neutrinos can have a much smaller number of measuring channels than of a optical Cherenkov detector with the same effective volume.

The possibility of acoustic detection of high-energy neutrinos and the energy detection threshold depend actually on the following factors: (1) the amplitude and the other parameters of acoustic signal caused by the interaction of a neutrino or other UHE particle with water, (2) the characteristics of ambient acoustic noise in a given water basin, and (3) the hardware and software capabilities for the detection and separation of the signals from ambient acoustic noise.

In this paper we review the present status and the first results of our activities towards the development of an acoustic neutrino detector in the Lake Baikal.

\section{Acoustic signal caused by the interaction of a neutrino in Baikal water}

The pressure $P(\mathbf{r}, t)$ of an acoustic signal from a cascade of relativistic particles in a point $\mathbf{r}$ and time $t$ is described by a wave equation with a so called "thermo-acoustic" source term:

$c_{s}^{2} \rho \nabla\left(\frac{1}{\rho} \nabla P\right)-\frac{\partial^{2} P}{\partial t^{2}}=-G \frac{\partial^{2} q(\mathbf{r}, t)}{\partial t^{2}}$

where $q(\mathbf{r}, t)$ is the energy deposition density along the cascade, $\rho$ the water density, $c_{s}$ is the adiabatic and non-dispersive speed of sound, $G$ is the Gruneisen parameter:

$G=\frac{\alpha}{c_{s}^{2}} C_{p}$

where $\alpha$ is the thermal expansion coefficient, and $C_{p}$ is the heat capacity. The acoustic pulse intensity at different sites and depths in a basin is given by the dimensionless Gruneisen parameter which value depends on the water temperature, its hydrostatic pressure and salinity. The depth dependence of $c_{s}$ in a vertically stratified medium (left hand plot on Fig. 1) leads as well to refraction effects for the wave-front discussed further in Section 3.

The Gruneisen parameter and therefore the acoustic signal strength increases with salinity, temperature and pressure, the last increases with depth. Examples of the depth dependence of $G$ are given in Fig. 1 (right) for Mediterranean Sea and Lake Baikal conditions. Due to the high salinity and the extraordinary warm temperature of water at large depth, close to $13{ }^{\circ} \mathrm{C}$ in Mediterranean Sea, the Gruneisen parameter is by a factor of 10 larger than that in fresh and cold Lake Baikal water, where in the deep zone the water temperature is $1.5-2^{\circ}$ larger than the temperature of maximum density at corresponding depth. This is a rather unfavourable condition for acoustic neutrino detection in the Lake Baikal because the expected strength of the acoustic emission should be by the same factor of 10 less compared with the Mediterranean Sea. Nevertheless, the possibility of acoustic detection of UHE neutrino and energy threshold of an array depend not only on signal amplitude but as well on absorption, ambient noise and its reduction efficiency, on signal processing techniques, etc.

The solution of Eq. (1) for pressure $P(\mathbf{r}, t)$ at the point $\mathbf{r}$ and time $t$ of acoustic signal from a cascade of relativistic particles is given by Kirchhoff integral:

$P(\mathbf{r}, t)=\frac{\alpha}{4 \pi C_{p}} \int \frac{d V^{\prime}}{\left|\mathbf{r}-\mathbf{r}^{\prime}\right|} \frac{\partial^{2}}{\partial t^{2}} q\left(\mathbf{r}^{\prime}, t-\frac{\left|\mathbf{r}-\mathbf{r}^{\prime}\right|}{c_{s}}\right)$.

The crucial point for the computation of this integral is a model for the energy deposition along the cascade, hence to get parameters of acoustic signal one should study the development of UHE underwater showers. Since experimental data on the interactions of such high energy particles do not exists it is necessary to use a theoretical model for the shower or to simulate it. In the pioneering papers of Askaryan et al. [9] and Learned [10], rather simple models of instantaneous heating of water volume were used. Now such studies rely mostly on Monte Carlo simulations and/or extrapolations from low-energy experimental data. The most extensive ultra high energy simulation program which has so far been developed is CORSIKA [13]. Originally this program has been used only for the simulation of cosmic ray air showers. In the paper of Ref. [14] CORSIKA was adopted to calculate showers produced by neutrino interactions in sea water at energies up to $10^{21} \mathrm{eV}$. Since the salt component of the water has a negligible effect on the signal we used the CORSIKA parametrization of the ACoRNE Collaboration [14] to calculate the energy deposition density $q(\mathbf{r}, t)$ for Baikal water.

Fig. 2 illustrates the distribution of energy deposition density $q(\mathbf{r}, t)$ in Baikal water. The resultant acoustic signal at any point is the sum of contributions from all points of the shower with corresponding amplitudes and time delays. Number of shower sample points versus sound propagation time from a shower point to a point of observation located in the energy maximum plane

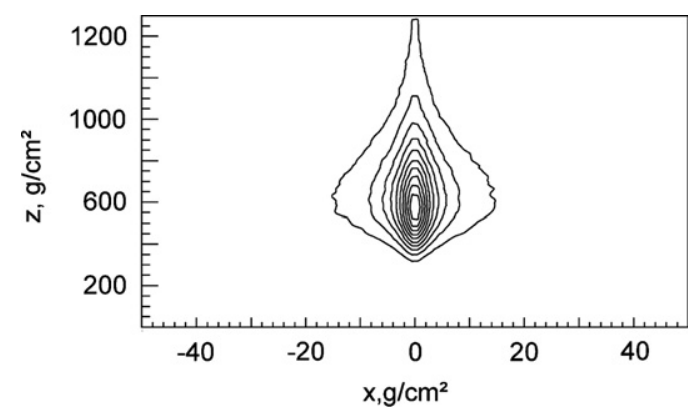

Fig. 2. Distribution of energy deposition density $q(\mathbf{r}, t)$ in Baikal water for shower with an energy $10^{20} \mathrm{eV}$. The closer to the figure center-the higher energy deposition density.
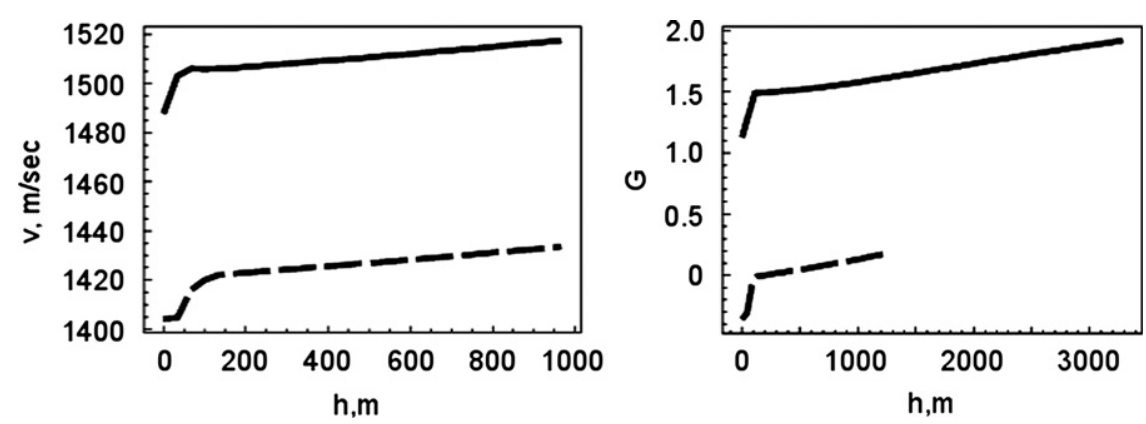

Fig. 1. Depth dependence of Gruneisen parameter $G$ (right) and speed of sound $c_{s}$ (left) for Mediterranean Sea (solid line) and Lake Baikal (dashed line). 
(the plane normal to shower axis where maximal energy of a shower is released) is shown on Fig. 3.

Fig. 4 shows the time profile and frequency spectrum of an acoustic pulse at the distance of $1 \mathrm{~km}$ from a neutrino induced cascade with energy $10^{20} \mathrm{eV}$ at $1 \mathrm{~km}$ depth in Baikal water, as simulated with our MC. Taking into account the non-uniform energy deposition density in the shower calculated with the MC code [14] results in a wider pulse spectrum and a shift to lower frequencies compared with the classical papers $[9,10]$.

Fig. 5 shows a very steep dependence of the peak pressure in the acoustic pulse with the angle from the energy maximum plane at $1 \mathrm{~km}$ from the shower. Due to the slow decrease of the pulse amplitude with distance and its sharp angular dependence, the optimal distribution of antennas for an acoustic neutrino detector should be quasi two-dimensional, in contrast to the three-dimensional arrangement of optical detectors in an underwater Cherenkov neutrino telescope. In other words, the hydrophones should be positioned on the same plane, predominantly on a horizontal

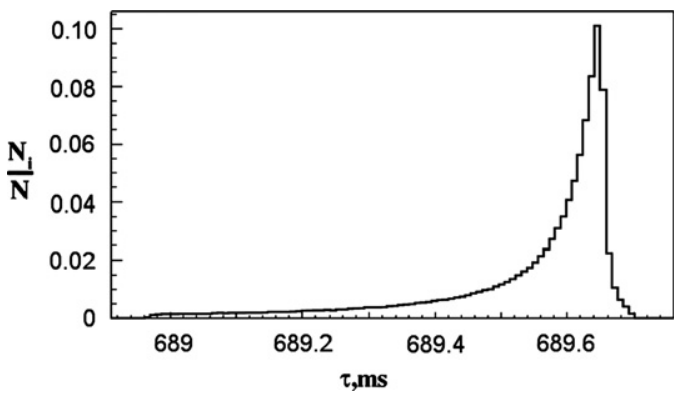

Fig. 3. Distribution of the propagation time from shower sample points to the observation point located on the energy maximum plane for a $10^{20} \mathrm{eV}$ shower.
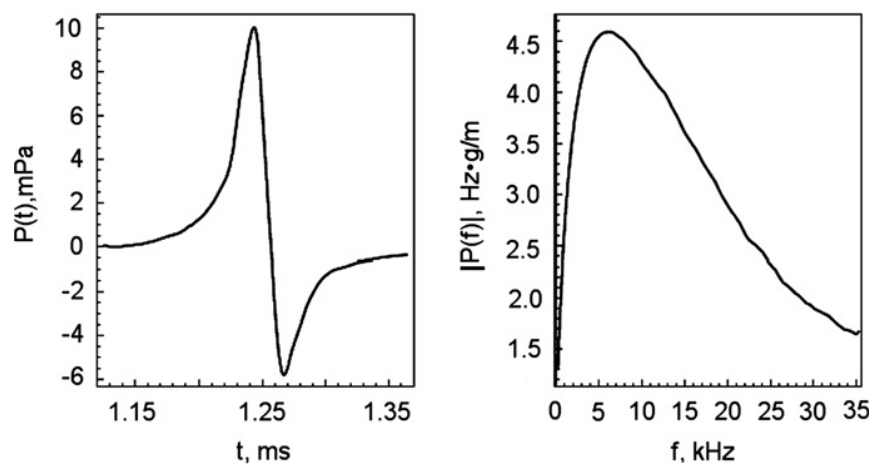

Fig. 4. Time profile and frequency spectrum of an acoustic pulse in the energy maximum plane at $1 \mathrm{~km}$ distance from the axis of the neutrino induced shower of energy $10^{20} \mathrm{eV}$, at $1000 \mathrm{~m}$ depth in Baikal water.

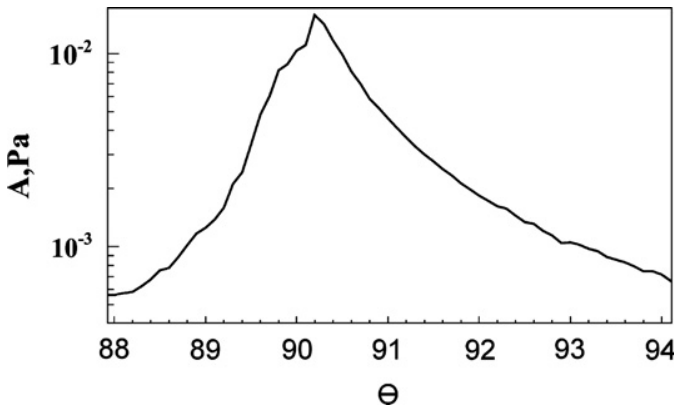

Fig. 5. Peak pressure of the acoustic pulse versus angle from the energy maximum plane at $1 \mathrm{~km}$ from a shower. plane-or close to a plane distribution, since for practical reasons it is rather difficult to obtain a strictly horizontal array geometry.

\section{Effect of refraction on the propagation of acoustic signals}

The dependence of the sound speed with the water depth leads to refraction phenomenon, i.e. distortion of the signal trajectories. True direction reconstruction of a source position can be performed via inverse traceability in the geometrical acoustic approximation. The algorithm is realized by an iterative procedure to determine the direction of the ray from the detector point location to the source point location with a given accuracy. This approach has been proposed in detail by Boyles [12]. In good approximation the speed of sound in the Lake Baikal starting from depth $100 \mathrm{~m}$ increases linearly with water depth:

$c_{s}=1418.96+0.01536 H$

where $H$ is depth in meters and $c_{s}$ in $\mathrm{m} / \mathrm{s}$.

Fig. 6 shows the effect of refraction on the trajectories of acoustic signals recorded by a detector located at depth of $200 \mathrm{~m}$ in the Lake Baikal. It is clearly seen, that signals with arrival angles at the detector from $0^{\circ}$ up to $131^{\circ}$ zenith may originate from sources located at the lake surface, and some even far away from the detector (taking the depth of the lake of about $1400 \mathrm{~m}$ into account).

\section{Background for acoustic neutrino detection on Lake Baikal}

The feasibility of acoustic neutrino detection at a site depends on some traditionally investigated statistical characteristics of ambient noise like: the acoustic noise floor in the relevant bandwidth and its variations, the noise power spectral density and the slope of spectra. In addition, the sensitivity of an acoustic particle detector depends to a large extent on the density and the distribution of sources producing "neutrino-like" bipolar pulses, and on the rate of such background signals.

To study noise from a wide range of sources in the Lake Baikal we have constructed an autonomous hydro-acoustic recorder with two input channels [15] and a digital hydro-acoustic module with four input channels, arranged on the corners of a regular tetrahedron, with an edge length of $1.5 \mathrm{~m}$ [16]. Long term monitoring of ambient noise with the instruments shows that noise power spectral density in the Lake Baikal decreases with a slope of spectra of about 5-6 dB/octave on average (see Ref. [15] for details). Noise level in the lake mostly depends on surface conditions because of weak attenuation of high-frequency acoustic waves in fresh water. In stationary and homogeneous meteorological conditions the RMS of the integral noise power in the relevant frequency range $5-20 \mathrm{kHz}$ reaches levels as low as $5 \mathrm{mPa}$ as it is seen from Fig. 7 .

To determine the properties of ambient noise as the background against which neutrino induced cascades should be detected,

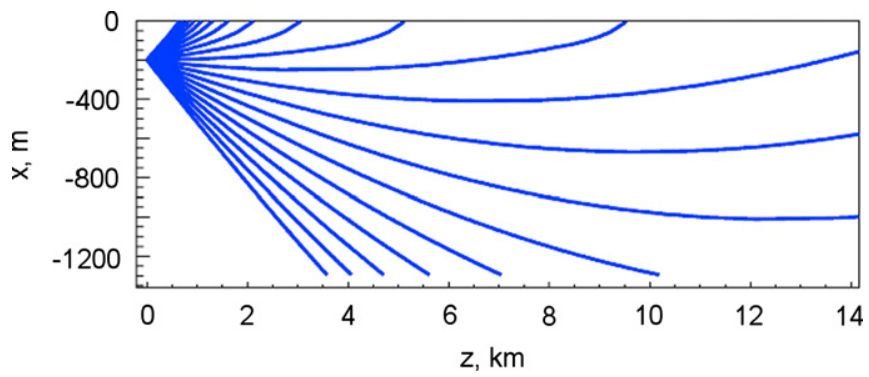

Fig. 6. Trajectories of acoustic propagation caused by refraction in the Lake Baikal, for a sensor at $200 \mathrm{~m}$ depth. 


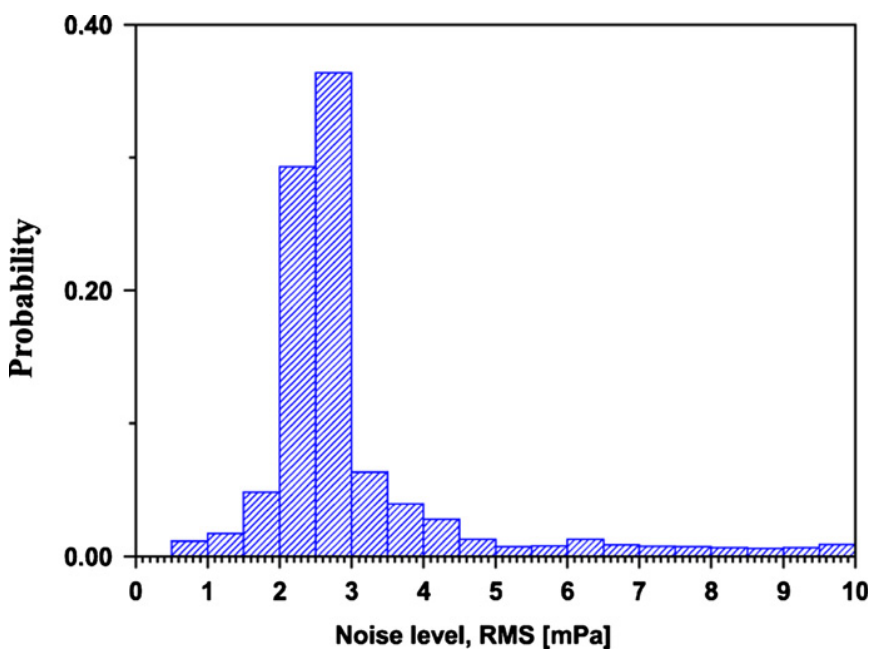

Fig. 7. RMS of the integral noise power in the Lake Baikal in the relevant bandwidth $5-20 \mathrm{kHz}$.

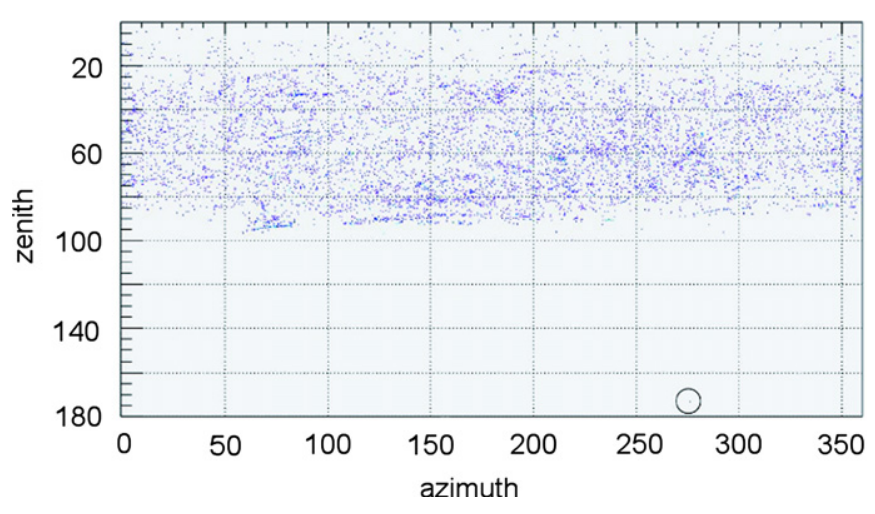

Fig. 8. The distribution of reconstructed zenith versus azimuthal angle for events with bipolar pulses.

we investigated the amplitude-time characteristics of Baikal noise in the high-frequency spectral region. Several years of study of the acoustic noise have shown, that the background for the acoustic detection of high-energy showers is primarily represented by transient noise with small pulse durations $[15,17]$.

Taking into account that the main part of background sources (waves, wind, rain, ships, biological sources and so on) are concentrated near the surface of the basin, we have installed a hydro-acoustic module with four input channels at a depth of $150 \mathrm{~m}$ (at one of the moorings of NT200+) for long term monitoring of the ambient noise. In this way we watch acoustically the water body from top-down. Fig. 8 shows for reconstructed acoustic events the distribution of azimuth versus zenith angles. For all of the about 7000 reconstructed bipolar acoustic events we find arrival directions of zenith $<100^{\circ}$, i.e. they originate from sources located in the top zone of the Lake (note: pulses slightly below horizon with zenith $>90^{\circ}$ are expected due to refraction). Only a single event is reconstructed as occurring in the deep part of the lake. This event has a zenith angle of $176^{\circ}$ and is clearly isolated in Fig. 8. The corresponding four hydrophone signals are shown in Fig. 9.

More information about the position and the properties of sources of neutrino-like pulses will be available with a grid of rather compact acoustic detectors arranged at shallow depths (100-200 $\mathrm{m}$ for Lake Baikal). An R\&D program to develop
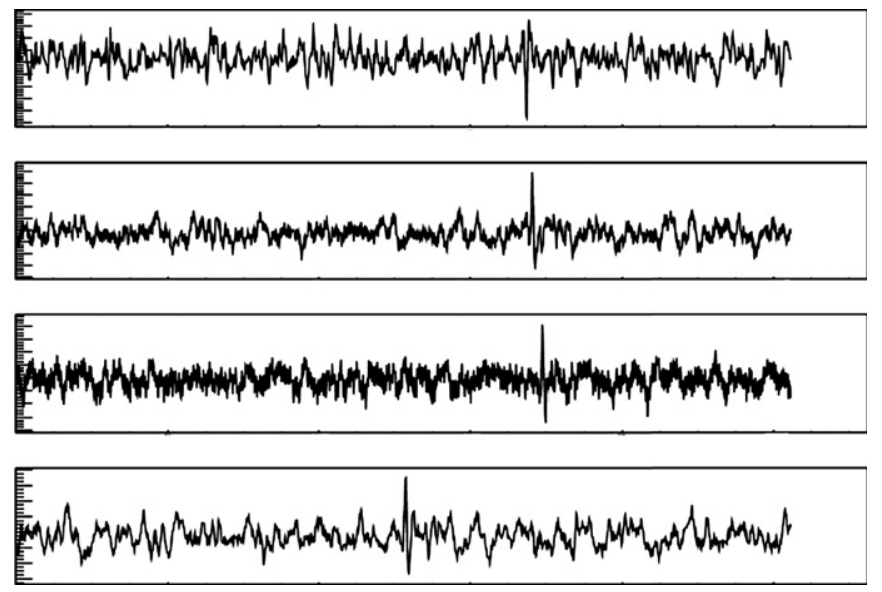

Fig. 9. The acoustic event reconstructed as upward "neutrino-like" (see Fig. 8), raw pulse traces (channels 1-4).

a prototype acoustic detector for high energy neutrinos has been started in 2009. The main features of the project are presented in the next section.

\section{A prototype acoustic detector for high energy neutrinos}

The idea of acoustic neutrino detection is in that cascades, generated by neutrino interactions in water, should produce bipolar acoustic transients. The expected pulse duration is of the order of several tens of microseconds. Most of the acoustic signal energy is concentrated within a disk, the axis of which coincides with the axis of the cascade. This feature can be used to search for such signals.

Devices capable of detecting definitely shaped signals and to interpret them in the terms of plane wave, generated by a quasilocal source, are expedients to use as an elementary unit of the acoustic neutrino detector. We will name the devices-Acoustic Sensor Module (ASM).

During the upcoming years we intend to deploy in the Lake Baikal a prototype acoustic neutrino detector. It will consists of a minimum of three strings. Each string will have four ASM at depths $125,150,175$ and $200 \mathrm{~m}$. To extract weak "neutrino-like" pulse signals from the background and to reconstruct a direction on a pulse source each ASM will be equipped with antenna with four hydrophones. The optimum distance between the hydrophones is defined by the condition that it must surely exceed several wavelengths of the expected signal, but, on the other hand, it should not be too long in order to minimise the number of background transients captured by the "time window" (see below). So, we arranged the hydrophones in a pyramid-like geometry with equal distances between the hydrophones of about $1.5 \mathrm{~m}$ (Fig. 10).

As the sensors we use special waveguide hydrophones (Fig. 10) which have a rather high and flat sensitivity in frequency bandwidth $10-30 \mathrm{kHz}$, where a large part of the acoustic energy of the pulse from the shower is concentrated. Waveguide hydrophones have a high sensitivity in a cone of the order of $90^{\circ}$ only and rather small sensitivity in the opposite direction (Fig. 11). The latter allows to decrease the influence of the background produced by near-surface sources.

Fig. 12 gives a schematic view of the ASM. Preamplifiers with $20 \mathrm{~dB}$ gain factor are integrated with sensors. The first preamplifier stage is based on chip AD743 with FET-head end, AD743 have a ultra low noise current $6.9 \mathrm{fA}$. The second preamplifier stage is based on the OPA1632DGN chip—a full differential amplifier. At 
this stage single-ended signal is transduced to differential-mode signal for anti-jamming of transmission via signal cables. For further amplification of the signals we use audio operational amplifier PGA2500 with differential output and software programmable amplification. In the range $0-3 \mathrm{kHz}$, the relative amplification is lowered by $40 \mathrm{~dB}$ per octave in order to suppress low frequency noise. High-frequency roll-off starts at $50-60 \mathrm{kHz}$. Bandpass flatness in the band $4-50 \mathrm{kHz}$ is less then $3 \mathrm{~dB}$. The signals are digitized by the 24 -bit/192 $\mathrm{kHz}$ over sampling Texas Instruments four channels sigma delta ADC-PCM4204. To decrease raw data flow to shore, the data are pre-processed in situ by the digital signal processors OMAPL138.

For inter communication between ASM and the socket center of the detector a PICOe-Atom computer is used. Fig. 13 shows the communication scheme of the detector. A LAN controller with

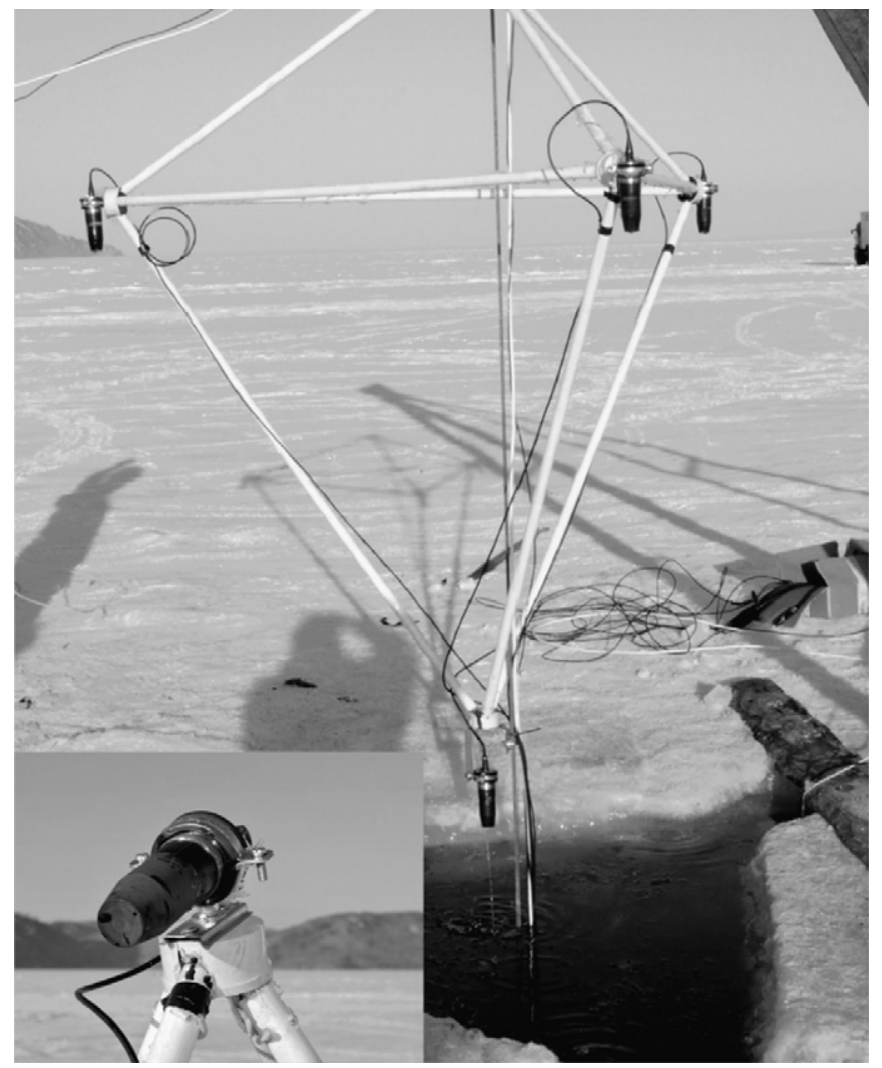

Fig. 10. The antenna of the Acoustic Sensor Module with four waveguide hydrophones (in left bottom corner) arranged in a pyramid-like geometry.
N ports with Power over Ethernet support allows to use same cables for signal and for power thus reducing the number of connections needed.

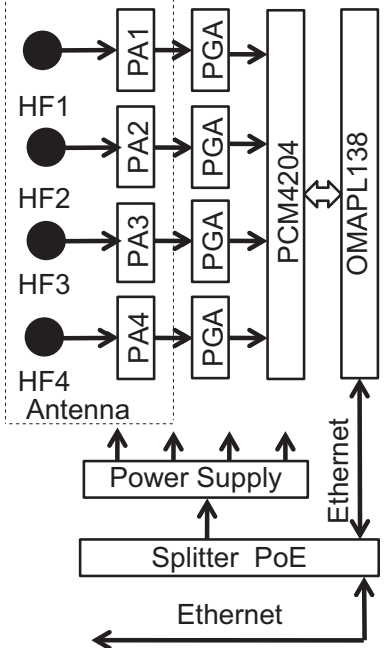

Fig. 12. Schematic view of the Acoustic Sensor Module. Preamp-integrated in hydrophone preamplifier, $P G A 2500$ - differential amplifier with programmable gain coefficient, PCM4204 - four channels 24 bit ADC, SOM-compute system based on XOMAPL138 module, Splitter PoE-splitter of data and power supply.

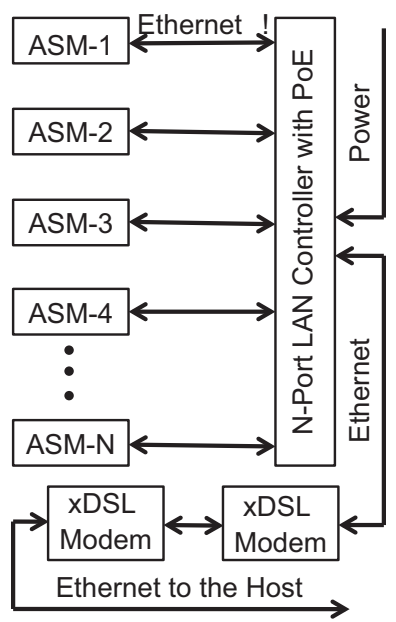

Fig. 13. Schematic view of the Data Acquisition System of the prototype acoustic detector for high energy neutrinos. ASM-Acoustic Sensor Module, N-Port LAN Controller with PoE-a LAN controller with N ports with Power over Ethernet support. VC-201-megabit modem.
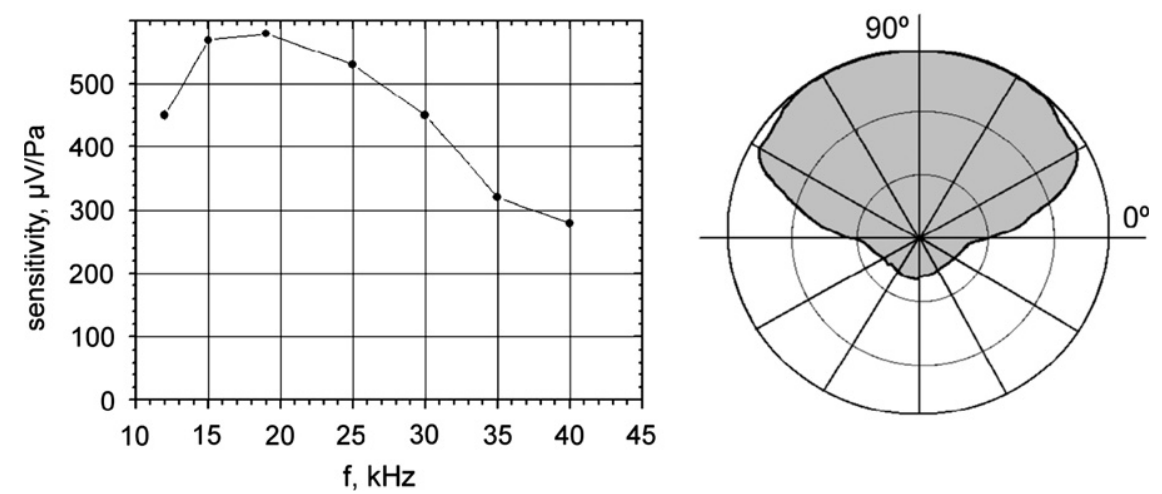

Fig. 11. Gain-frequency characteristics (left) and acoustic pattern (right) of waveguide hydrophones. 


\section{In situ algorithm for search of "neutrino-like" acoustic signals}

The detection algorithm has two main tasks. The first is to detect signals with level higher than the given threshold. The second is to dynamically correct detection threshold relying on current statistical characteristics of ambient sound. The program distinguishes the following properties of the signals: their coordinates within time series, their duration, the number of periods, the maximal amplitude etc. Transients are classified by these parameters and the information about them is stored in corresponding dynamic arrays.

- Filtration procedure: Events satisfying the condition $\Delta t_{i j}<k t_{\max }$ for every $\Delta t_{i j}=t_{i}-t_{j}$ will be accepted. Here $t_{i}$ is the signal detection time in i-th acoustic channel, $t_{\max }=L / c_{s}$ is the time of sound propagation between hydrophones, $L$ is the distance between two neighboring hydrophones, $c_{s}$ the sound velocity at the depth where the antenna is placed, and $k=1.2$ is the tolerance for $\Delta t_{i j}$ estimation.

- Direction estimation:

At the final step, we obtain direction angles $\theta, \phi$ by minimisation of a functional $S(\theta, \phi)$ :

$S=\sum_{i=1}^{4}\left[t_{i}-\tilde{t}_{i}\right]^{2} \frac{1}{\sigma_{t_{i}}^{2}}, \quad t_{i}=t_{0}+\left(r_{i}-r_{0}\right) n$

where $t_{i}$ is the expected start point of a signal in time series; $\tilde{t}_{i}$ the measured start point; $r_{0}$ the pyramid centre position in the chosen system of coordinates; $t_{0}$ the propagation moment of a sound wave through a point $r_{0} ; \sigma_{t_{i}}^{2}$ the measurement deviations of time coordinates; $n(\theta, \phi)$ the vector normal to the acoustic front.

It was developed a hydrophone based bipolar signals transmitter for calibration purposes. The transmitter was installed underwater at a distance of $50 \mathrm{~m}$ from the ASM antenna. About 2000 events were recorded and processed further by a correlation analysis procedure. Fig. 14 shows the distribution of acoustic signals versus zenith and azimuth angles during the measurement (calibration pulses are shown on superimposed histogram). The RMS of reconstructed angles are $\sigma_{\phi} \approx 1.5^{\circ}$ and $\sigma_{\theta} \approx 0.5^{\circ}$.

\section{Summary and outlook}

Acoustic neutrino detection in the Lake Baikal and in other natural reservoirs is far from being trivial due to high level of background noise. Comparison of results of acoustic pulse characteristic calculations and properties of noise in Lake Baikal shows that energy threshold for neutrino detection in the lake should be of order $10^{19}-10^{20} \mathrm{eV}$. Taking into account that sources of background including neutrino-like short pulses are concentrated at near-surface zone we suggest an acoustic detector design that is based on the deployment of a grid of Acoustic Sensor Modules with rather compact antennas arranged at shallow depths (100-200 m for Lake Baikal). These antennas select signals from the bottom of the lake. R\&D of a prototype cluster of an acoustic neutrino

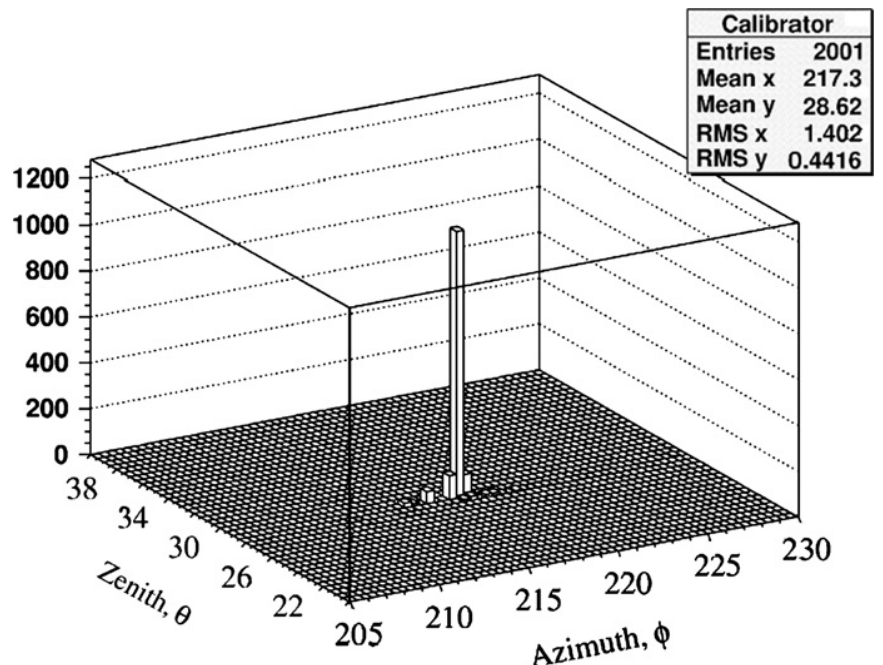

Fig. 14. A distribution of reconstructed angles for the acoustic pulses generated by the pulse simulator.

telescope in the Lake Baikal has been started this year. In March 2011 we plan to install the first mooring with four second generation Acoustic Sensor Modules equipped by antenna with four hydrophones.

\section{Acknowledgements}

This work was supported in part by the Russian Ministry of Education and Science (GK 02.740.11.0018, GK 02.518.11.7158, P133, P878, P1146, P1242, P2504, RNP 2.2.1.1.1483, RNP 2.1.1.1539, RNP 2.2.1.1.5901, REC “BAIKAL"), by the German Ministry of Education and Research, by the Russian Found for Basic Research (Grants 07-02-00791, 08-02-00432, 08-02- 00198, 09-02-10012, 10-02-10000, 09-02-00623, 09-02-12295).

\section{References}

[1] M.A. Markov, On high energy neutrino physics, in: Proceedings of the 1960 Annual International Conference on High Energy Physics, Rochester, 1960, p. 578

[2] V. Aynutdinov, et al., Astropart. Phys. 25 (2006) 140.

[3] I. Belolaptikov, et al., Astropart. Phys. 7 (1997) 263.

[4] A. Achterberg et al., Astropart. Phys. 26 (2006) 155.

[5] J. Carr, ANTARES Collaboration, Nucl. Instr. and Meth. A 588 (2008) 80.

[6] R. Wischnewski, For the Baikal Collaboration, in: Proceedings of the 30th ICRC Conference, Merida, Mexico, 2007, arXiv:0710.3063.

[7] V. Aynutdinov, et al., Nucl. Instr. and Meth. A 602 (2009) 14

[8] G.A. Askaryan, Atomnaya Energiya 3 (1957) 152.

[9] G.A. Askaryan, et al., Nucl. Instr. and Meth. 164 (1979) 267.

[10] J.G. Learned, Phys. Rev. D 19 (1979) 3293.

[11] C.S. Clay, H. Medwin, Acoustical Oceanography, Wiley, New York, 1977.

[12] C.A. Boyles, Acoustic Waveguides: Applications to Oceanic Science, Wiley, New York, 1984

[13] D. Heck, et al., CORSIKA: A Monte Carlo Code to Simulate Extensive Air Showers, Karlsruhe Report FZKA 6019 〈http://www-ik.fzk.de/corsika〉.

[14] S. Bevan, et al., Astropart. Phys. 28 (2007) 366

[15] V. Aynutdinov, et al., Acoust. Phys. 52 (2006) 495

[16] V. Aynutdinov, et al., Nucl. Instr. and Meth. A 604 (2009) S130.

[17] V. Aynutdinov, et al., Int. J. Mod. Phys. A 21S1 (2006) 117. 